\title{
Comunidades de aves y lepidópteros diurnos y las relaciones entre ellas en bosque nuboso y cafetal de Finca Santa Maura, Jinotega
}

\author{
Marvin Tórrez, Wayne Arendt y Jean Michel Maes ${ }^{* * *}$
}

Recibido: abril de 2013 / Aceptado: junio de 2013

Para evaluar la diversidad de aves y mariposas ninfálidas visitamos la Estación Biológica Juan Roberto Zarruk en Jinotega, donde colectamos datos en todos los hábitats presentes en la finca. Obtuvimos 123 especies de aves y 29 especies de ninfálidos. El hábitat con mayor riqueza para aves fue el cafetal y para ninfálidos el tacotal. Entre las especies más comunes encontramos especies dependientes de bosque, mostrando que el paisaje aún alberga biodiversidad de importancia para la conservación. Los cafetales, dado que están inmersos en una matriz compleja con remanentes de bosque, tienen en su interior especies de importancia para la conservación, respaldándose la idea de que las prácticas agroecológicas y de protección de los remanentes de bosque tienen un efecto positivo en este paisaje, permitiéndonos poder seguir midiendo el pulso de la biodiversidad en esta área.

Palabras clave: agropaisaje / ninfálidos / bosque nuboso / especies dependientes

\section{Introducción}

La conservación de los bosques y su equilibrio con la agricultura es un tema de vital importancia en Mesoamérica (Morales, Ferguson \& García-Barrios, 2007). Se ha demostrado que las prácticas agrícolas en los trópicos causan pérdida de biodiversidad nativa y dependiente de bosque (Walter, Bobo, Sainge, Fermon \&

* Universidad Centroamericana, Rotonda Rubén Dario 150 m. al oeste. Apdo. 69, Managua, Nicaragua. Correo electrónico: mtorrez@ns.uca.edu.ni

**Instituto Internacional de Dasonomía Tropical, San Juan, Puerto Rico y Servicio Forestal de los Estados

Unidos, Estación de Investigaciones, Sabana, HC 2, Buzón 05, Luquillo, PR 00773, Estados Unidos.

***Museo Entomológico de León, de las Oficinas Centrales de Unión Fenosa, 30 varas arriba, León, Nicaragua. 
Mühlenberg, 2005; Steffan-Dewenter et al., 2007). Sin embargo, esta pérdida puede ser mitigada a largo plazo con un manejo adecuado de cultivos y remanentes de bosque (Priess et al., 2007).

En Nicaragua el papel que juegan los distintos hábitats dentro de los paisajes ha sido evaluado en diversos estudios (Harvey et al., 2006; Pérez et al., 2006; Vílchez et al., 2007) y estos estudios han tenido el objetivo de entender los patrones de diversidad de la fauna, además de demostrar cómo los paisajes dominados por actividades agrícolas (Agropaisaje), complementan la falta de hábitats homogéneos en pro de hábitats fragmentados que permiten un componente de diversidad significativo (Walter et al., 2005).

El presente estudio muestra el papel de los diversos hábitats dentro de un agropaisaje dominado por el cultivo de café en la zona de vida del bosque nuboso. Estudios acerca del valor ecológico de los cafetales en aves y mariposas han sido realizados en Latinoamérica con anterioridad (Mas \& Dietsch, 2004; Philpott et al., 2008), por lo que este estudio aportará información importante a nivel nacional.

\section{Metodología}

\subsection{Sitio de estudio}

El estudio se realizó en la Estación Biológica Juan Roberto Zarruk (EBJRZ) ubicada

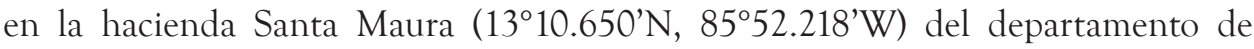
Jinotega. El área total de la hacienda tiene alrededor de 650 hectáreas, las que están destinadas al cultivo de café, con remanentes de bosque distribuidos a lo largo del paisaje. La altura promedio en la estación varía entre los 1100 a los 1250 msnm con una precipitación anual promedio de $2500 \mathrm{~mm}$. Es parte del sistema de áreas protegidas de Nicaragua, específicamente de la reserva natural Datanlí-El Diablo, clasificada como bosque húmedo premontano por su zona de vida (Holdridge, 1996). Asimismo, es parte del bioma zoogeográfico de las tierras altas de México y el norte de Centroamérica (Stotz, Fitzpatrick, Parker \& Moskovits, 1996). En esta reserva se protegen especies características del bosque húmedo del norte de Centroamérica (Howell \& Webb, 1995).

El sitio de estudio fue seleccionado para formar parte del proyecto “Conservación y Turismo Sostenible en Cuencas Críticas" (Bauer \& Arendt, 2007), por lo que previamente fueron evaluados los hábitats dentro de la zona, tomando en cuenta la estructura horizontal y vertical (porcentaje de cobertura en dosel, presencia de arbusto y lianas, altura de dosel, DBH) y dividiéndolos según su historia natural y el tipo de bosque según Louman y Quirós (2001). La terminología se adaptó a estudios anteriores relativos a la ecología de paisaje (Harvey et al., 2006; Vílchez et al., 2007), según lo cual se caracterizaron cuatro hábitats: Bosque cerrado, Bosque secundario joven (tacotal), Bosque de ribera y Cafetal.

El sitio de estudio fue visitado cinco veces entre 2009 y 2010. Las visitas de 2009 fueron en los meses de febrero, marzo, julio y septiembre, y en 2010 fue en el mes de abril. La cantidad de puntos visitados en las distintas ocasiones están resumidos en el Cuadro 1. Los puntos de conteo de aves fueron visitados durante las 
cinco entradas, mientras que los de mariposas únicamente se visitaron cuatro veces (no se realizó la visita en el mes de septiembre de 2009).

\subsection{Método de conteo de aves}

Se detectaron aves en puntos de conteo de radio fijo ubicados en los distintos hábitats presentes en la hacienda. Cada punto tuvo una distancia promedio de 200 m. Los puntos se realizaron entre 0540-0930 hrs cuando las condiciones de clima resultaron favorables, suspendiendo por lluvia o exceso de viento principalmente. Se identificaron las aves utilizando guías de campo para México y el norte de Centroamérica de Howell y Webb (1995), y de Costa rica (Stiles \& Skutch, 1989). Los gremios alimenticios se determinaron según la Guía de Aves de Costa Rica (Stiles \& Skutch 1989) y otras publicaciones (Willson, Porter \& Condit,1982; Remsen, Hyde \& Chapman, 1993; Poulin, Lefebvre \& McNeil, 1994; Verea \& Solórzano, 2001; Pearman, 2002; Van Bael, Bichier, Ochoa \& Greenbeg, 2007). El uso de múltiples referencias nos permitió estandarizar las categorías de gremio, así como llenar vacíos de información en aquellas especies no determinadas por las guías. Finalmente, los gremios que se determinaron para el presente estudio fueron los siguientes: insectívoro buscador, frugívoro, nectarívoro e insectívoro. Gremios tales como omnívoro o carnívoro no fueron parte de estos análisis pues una prueba a priori no demostró que tuvieran alguna relación significativa entre los distintos hábitats.

\subsection{Captura de mariposas}

Se colocaron trampas de frutas a $25 \mathrm{~m}$ una de otra en los mismos sitios donde se realizaron los puntos de conteo de aves. Las trampas eran cebadas en la mañana a las $0700 \mathrm{hrs}$ y revisadas a las $1500 \mathrm{hrs}$. Las mariposas eran introducidas en sobres de papel, marcadas con códigos individuales y llevadas al Museo Entomológico de León, donde se almacenaron. El número de trampas de frutas por hábitat fue de siete.

\section{Cuadro 1. Número de puntos de muestreo (aves) y trampas usados durante el estudio}

\begin{tabular}{ccc}
\hline \hline Taxa & & Puntos/Trampas \\
\hline Aves & & 34 \\
& Bosque & 109 \\
& Café & 22 \\
& Ripario & 49 \\
& Tacotal &
\end{tabular}

Bosque 


$\begin{array}{cc}\text { Café } & 10 \\ \text { Ripario } & 10 \\ \text { Tacotal } & 10\end{array}$

\section{Análisis de los datos}

Se realizaron comparaciones de los tipos de hábitats presentes en la zona de estudio usando pruebas de ANOVA, para lo cual se emplearon modelos lineales mixtos (Di Rienzo, Macchiavelli \& Casanoves, 2010) desde el programa InfoStat (Di Rienzo et al., 2011). Esto nos nos permitió seleccionar el mejor modelo para nuestros datos.

A posteriori se realizaron comparaciones de fischer que nos ayudaron a observar cuáles grupos presentaban las diferencias significativas. Realizamos regresiones lineales desde el programa InfoStat (Di Rienzo et al., 2011) que nos permitieron saber cómo los grupos de gremios en los hábitats se asocian y encontrar cuáles se asocian mejor con la riqueza de especies de las comunidades (Schulze et al., 2004; Harvey et al., 2006).

Para el grupo de las mariposas, antes de proceder con los análisis se realizó una estandarización de media ponderada por trampa por tiempo trabajado (Bouyer et al., 2007).

El cambio o reemplazo de especies se calculó con el Índice de Jaccard a través de Biodiversity Pro.

\section{Resultados}

Se lograron detectar a lo largo del estudio 1484 individuos en 123 especies de aves y 128 individuos capturados en 29 especies de mariposas (Cuadro 2). El hábitat con la mayor riqueza de aves fue el cafetal (92 especies), mientras que la mayor riqueza de mariposas se dio en el tacotal (18 especies). De las 123 especies de aves 20 fueron especies migratorias distribuidas de la siguiente manera: cafetal con 14 especies, seguido de tacotal con 10, ripario con 8 y bosque con 7 .

\section{Cuadro 2. Resultados totales de las observaciones y capturas de aves y mariposas}

\begin{tabular}{lllllll}
\hline \hline Taxa & Hábitat & Bosque & Tacotal & Ripario & Café & Total \\
\hline \multirow{2}{*}{ Aves } & Abundancia & 219 & 315 & 94 & 856 & 1484 \\
& Riqueza & 47 & 76 & 33 & 92 & 123 \\
\multirow{2}{*}{ Mariposas } & Abundancia & 29 & 51 & 26 & 22 & 128 \\
& Riqueza & 12 & 18 & 15 & 9 & 29
\end{tabular}

Nota: Para aves: bosque $(n=6)$; tacotal $(n=7)$; ripario $(n=4)$; café $(n=20)$. Para mariposas: bosque, tacotal, café $(n=7)$, ripario $(n=5)$. La disparidad de esfuerzo fue dada por la disponibilidad de hábitat en los distintos usos de suelo. 
Entre las especies de aves más detectadas sobresalen especies generalistas como Psilorhinus morio y Saltator atriceps, mientras que en mariposas sobresalen especies de hábitats boscosos como Yphthimoides renata Satyrotaygetis gigas (Cuadro 3).

Cuadro 3. Especies más comunes mostrando el porcentaje de presencia en el estudio con respecto a los totales

\begin{tabular}{llllllll}
\hline \hline & Bosque & Tacotal & Ripario & Café & Total & $\%$ \\
\hline & & & & & & $(\mathrm{n}=1484)$ \\
Psilorhinus morio & 11 & 8 & 3 & 90 & 112 & 7.5 \\
Saltator atriceps & & 25 & 1 & 61 & 87 & 5.9 \\
Henicorhina leucosticta & 25 & 32 & 17 & 8 & 82 & 5.5 \\
Basileuterus culicivorus & 26 & 13 & 8 & 6 & 53 & 3.6 \\
Basileuterus rufifrons & 1 & 3 & 7 & 41 & 52 & 3.5 \\
Dives dives & 1 & 3 & & 42 & 46 & 3.1 \\
Tolmomyias sulphurescens & 9 & 15 & 6 & 15 & 45 & 3.0 \\
Campylorhynchus zonatus & & 5 & & 39 & 44 & 3.0 \\
Saltator maximus & 1 & 10 & 1 & 31 & 43 & 2.9 \\
Phaethornis longirostris & 27 & 8 & 3 & 4 & 42 & 2.8 \\
\hline & & & & & & $(\mathrm{n}=128)$ \\
& & & & & & & \\
Yphthimoides renata & 6 & 6 & 5 & 5 & 22 & 17.2 \\
Satyrotaygetis gigas & 4 & 7 & 3 & & 14 & 10.9 \\
Archaeoprepona amphimachus & 4 & 4 & 1 & & 9 & 7.0 \\
Cyllopsis suivalenoides & & 7 & & 2 & 9 & 7.0 \\
Taygetis andromeda & 2 & 4 & 3 & & 9 & 7.0 \\
Pareuptychia metaleuca & 2 & 4 & 2 & & 8 & 6.3 \\
Smyrna blomfildia & 2 & 1 & 1 & 4 & 8 & 6.3 \\
Hermeuptychia hermes & & 1 & & 5 & 6 & 4.7 \\
Morpho helenor & 2 & 3 & 1 & & 6 & 4.7 \\
Dioristes tauropolis & & 4 & 1 & & 5 & 3.9
\end{tabular}

Al observar las especies según su índice de afinidad (Cuadro 4; Ilustración 1), observamos que los hábitats que se asocian en un mayor porcentaje son bosquetacotal-ripario, para ambos grupos: aves y mariposas. Sin embargo, a diferencia del grupo de las aves, el grupo de las mariposas muestra una similitud mayor de tacotalcafetal, que la que ocurre con las aves (Cuadro 4).

Cuadro 4. Similitud de Jaccard para mariposas

\begin{tabular}{llllll}
\hline \hline & & Bosque & Café & Ripario & Tacotal \\
\hline Aves & Bosque & $*$ & 16.5581 & 47.2843 & 50.5618 \\
& Café & $*$ & $*$ & 13.0526 & 29.889 \\
& Ripario & $*$ & $*$ & $*$ & 38.1418 \\
& Tacotal & $*$ & $*$ & $*$ & $*$
\end{tabular}




$\begin{array}{rlllll}\text { Mariposas } & \text { Bosque } & * & 30.9524 & 60.7595 & 49.2063 \\ \text { Café } & * & * & 30.137 & 43.3333 \\ \text { Ripario } & * & * & * & 40 \\ \text { Tacotal } & * & * & * & *\end{array}$

Nota: El valor del indice se muestra en este caso como porcentajes de especies compartidas.

Es evidente en la Ilustración 1 que el dendrograma separa al cafetal de los otros hábitats, demostrando que la riqueza de especies en este hábitat no muestra asociación alta con los hábitats de coberturas boscosas.

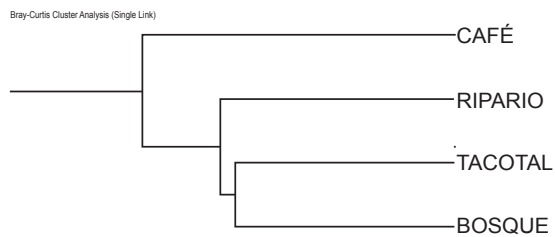

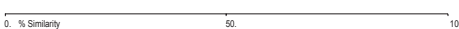
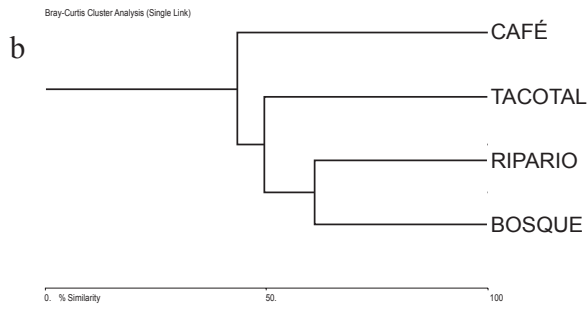

Ilustración 1. Dendrograma de similitud del índice de Jaccard, a) aves y b) mariposas.

La comunidad de aves presentó diferencias significativas tanto para las detecciones como para la riqueza $\left(\mathrm{P}<0.0001, \mathrm{~F}_{1,3}=8.8 ; \mathrm{P}=0.0154 \mathrm{~F}_{1,3}, 3.57\right)$, siendo el hábitat con mayor promedio de detecciones el cafetal y con menor el ripario. Para la comunidad de mariposas existió diferencia significativa para la riqueza de especies $\left(\mathrm{P}=0.05, \mathrm{~F}_{1,3}\right.$ 2.77) donde el tacotal y el ripario fueron los hábitats con mayor riqueza. Sin embargo, para las capturas de este grupo no hubo diferencias significativas $\left(\mathrm{P}=0.1370, \mathrm{~F}_{1,3} 1.93\right)$.
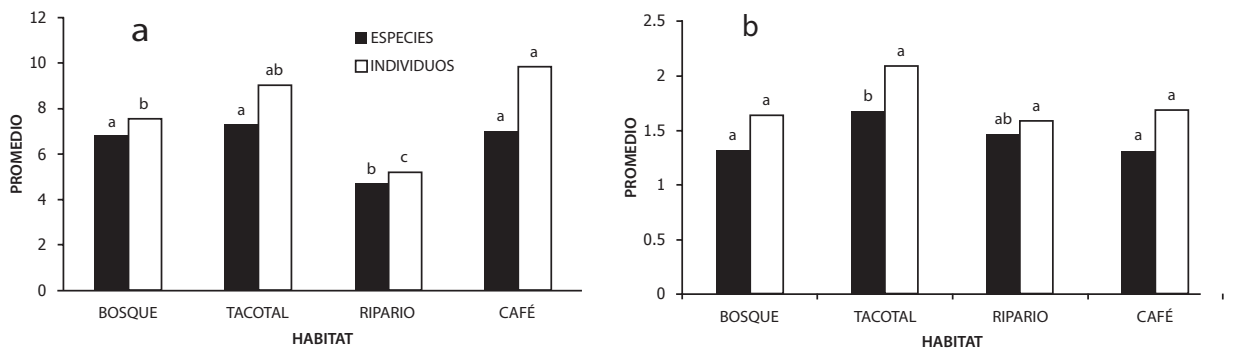

Ilustración 2. Comparaciones de las medias de abundancia y riqueza en (a) aves y (b) mariposas

$\mathrm{Al}$ analizar cómo se asocian la riqueza de especies con los distintos hábitats, los análisis de regresión lineal mostraron que la riqueza de especies de mariposa no tuvo correlación significativa con las riqueza de aves ni con los gremios de la 
comunidad de aves (Cuadro 5). Sin embargo, en la comunidad de aves el gremio de los nectarívoros fue el que mostró mayor número de correlaciones significativas con otros gremios y grupos, seguido de los insectívoros e insectívoros buscadores.

\section{Cuadro 5. Relaciones entre los gremios y las riquezas de aves y mariposas}

\begin{tabular}{lcccccc}
\hline \hline & Sp Aves & Sp Marip & Insec Busc & Frugívoro & Nectarívoro & Insectívoro \\
\hline Sp & -0.01 & & -0.11 & 0.03 & -0.04 & 0.00 \\
Mariposas & $(0.7295)$ & $\ldots$ & $(0.1382)$ & $(0.4797)$ & $(0.4031)$ & $(0.9832)$ \\
Insec & 0.22 & -0.13 & & 0.02 & 0.24 & 0.17 \\
Buscador & $(0.0309)^{*}$ & $(0.1146)$ & $\ldots$ & $(0.5080)$ & $(0.0251)^{*}$ & $(0.0611)$ \\
& 0.15 & 0.02 & 0.02 & & 0.12 & 0.16 \\
Frugívoro & $(0.0838)$ & $(0.5768)$ & $(0.5395)$ & $\ldots$ & $(0.1129)$ & $(0.0745)$ \\
& 0.22 & -0.02 & 0.24 & 0.23 & & 0.20 \\
Nectarívoro & $(0.0316)^{*}$ & $(0.4985)$ & $(0.0147)^{*}$ & $(0.0298)^{*}$ & $\ldots$ & $(0.0406)^{*}$ \\
& 0.45 & 0.0003 & 0.25 & 0.12 & 0.14 & \\
Insectívoro & $(0.0009)^{*}$ & $(0.9365)$ & $(0.0206)^{*}$ & $(0.1165)$ & $(0.1007)$ & $\ldots$
\end{tabular}

*Valores con correlación significativa, $p<0.05$.

Nota: Los valores corresponden al r2 y el valor de p se presenta entre paréntesis.

\section{Discusión}

Se ha logrado detectar durante el estudio aproximadamente el 59\% de la riqueza de aves que se conocen a la fecha en la zona (Hernández, Morales, Arendt \& Tórrez, 2009) y aproximadamente el $80 \%$ de las mariposas que se pueden capturar con trampas encontradas en este zona a la fecha (Maes, 2007). En aves las mayores detecciones fueron de aves generalistas (Cuadro 3). Esto se debe a que, en parte, más de la mitad de la finca está destinada a actividades humanas (café, vivienda, caminos). No obstante, es de resaltar que algunas especies de bosque, como H. leucosticta, estaban presentes en buen número, por lo que vemos cierto balance que es dado en parte por lo heterogéneo del hábitat. Asimismo, a pesar de considerarse vulnerable a los hábitats deforestados, esta especie pudo encontrarse en algunos puntos del hábitat cafetal. Sin embargo, éstas fueron observaciones a menos de $25 \mathrm{~m}$ del hábitat boscoso más cercano, demostrándose que aunque el cafetal no está reemplazando el hábitat para la especie sí provee refugio temporal o de forrajeo cuando el bosque está cerca. Esto refuerza la necesidad de agropaisajes heterogéneos en pro de hábitats homogéneos y perturbados (Harvey et al., 2006; Vílchez et al., 2007).

La alta presencia de aves migratorias en hábitats alterados (cafetal) o en regeneración temprana (tacotal) es concordante con otros estudios y publicaciones (Moore, Gauthreaux, Kerlinger \& Simons, 1995), por lo que su presencia en este hábitat no muestra algún patrón no estudiado antes. Además, encontramos en este hábitat evidencia de la existencia de algunas especies de aves migratorias que se encuentran en la lista roja de IUCN, tales como Dendroica fusca, la cual se encuentra categorizada como Vulnerable (IUCN, 2012). En cambio, la riqueza de mariposas 
en el cafetal fue menor, lo que en parte puede deberse a que las mariposas que se capturan con trampa de fruta tienen más afiliación con el bosque. Deducimos esto pues hemos observado en el cafetal otras especies pertenecientes a las familias Pieridae y Papilionidae, las cuales no son atraídas por la trampa, pero por motivos de rigurosidad en el método de muestreo no las hemos incluido en el estudio aunque las hayamos observado.

A grandes rasgos sobresale que el grupo de las mariposas muestra una clara tendencia a aumentar la riqueza de especies según aumenta la conservación del hábitat. Sin embargo, al observar detenidamente los datos, vemos cómo el hábitat cafetal no presenta una diferencia significativa con hábitats como el bosque (Ilustración 2). El porcentaje de especies que éstos comparten es menor que con otros hábitats (Cuadro 3), revelando que la calidad de las especies de aves que lo componen son diferentes, lo que constatamos al ver que especies menos sensibles a la perdida de hábitat (Stotz et al., 1996) son más comunes en café o ausentes en bosque (Cuadro 2).

Otro aspecto a resaltar es que las especies de mariposas no mostraron afiliaciones con los grupos de aves ni con su riqueza (Cuadro 5), lo que es interesante dado que se les considera buenos indicadores (Schulze et al., 2004; Bouyer et al., 2007). Sin embargo, debe de tomarse en consideración que la riqueza de aves en cafetal incluye especies que son generalistas y esto no significa que si la riqueza aumenta en este hábitat (Ilustración 2) sea por especies afiliadas en su calidad a hábitats de cobertura boscosa (Ilustración 1). Tomando como base un documento publicado por Maes (2013) logramos ver que algunas especies propuestas como indicadores positivos del género Cyllopsis spp están presente en nuestro estudio. Además podemos agregar otros, como los géneros Archaeoprepona spp y Satyrotaygetis spp, que aunque no son exclusivos de áreas conservadas, sí son más numerosas en estas últimas, y disminuyen conforme aumenta la alteración del hábitat (Cuadro 3).

El hecho que el grupo de nectarívoros muestre mayores relaciones con otros grupos en su riqueza de especies se debe en parte a que la familia Trochilidae es un grupo que muestra cierta relación a los hábitats conservados y las altitudes (Parra, Rahbek, McGuire \& Graham, 2011). Además, este grupo, en las zonas del Centro de Nicaragua presenta mayor riqueza de especies (Martínez-Sánchez, 2007), por lo que es un grupo de importancia ecológica en este ecosistema.

Las implicaciones de nuestros resultados para la conservación revelan lo importante de mantener remanentes de bosque cerca de áreas antropogenizadas para lograr, a nivel de paisaje, tener un equilibrio de especies tanto generalistas como especialistas. Las mejores prácticas no sólo tendrían un efecto positivo en la biodiversidad, sino también en las poblaciones humanas al mejorar la recepción de agua a nivel de cuenca, y aportar al equilibrio nutricional y ecológico de las poblaciones locales (Deckelbaum, Palm, Mutuo \& DeClerck, 2006). Además, desde el punto de vista de restauración de corredores es importante que los socios locales mantengan parches de bosque conectados, usando como marco leyes existentes como la Ley del Medioambiente (Asamblea Nacional de Nicaragua, 2008), donde se llama a la protección de las riberas (Art. 17), hábitat que es importante en la matriz del paisaje y con un gran portencial de conectividad. 


\section{Agradecimientos}

Queremos agradecer a los técnicos de campo, Henry López y Moisés Siles, por su apoyo en la fase de campo. Es de igual manera importante agradecer al Sr. Jerry Bauer por su apoyo a la conservación en Nicaragua y este proyecto en específico, a la ONG Paso Pacífico por apoyar en la logística del mismo y su trabajo en la zona, y a Don Jorge Chávez, propietario de la Finca Santa Maura, por su continuo aporte a la conservación en Jinotega.

\section{Referencias bibliográficas}

Asamblea Nacional de Nicaragua. (2008, 03 de abril). Ley de reformas y adiciones a la Ley 217, "Ley del medio ambiente y los recursos naturales. Managua, La Gaceta, (62).

Bauer, G. P. \& Arendt, W. J. (2007). Conservación y turismo en cuencas críticas (Conservation and sustainable tourism in critical watersheds). Participating Agency Service Agreement, USAID/Nicaragua and US Forest Service, USAID-PASA No. 524-P-00-00-07-00007-00. Unpl. Fact Sheet.

Bouyer, J., Sana, Y., Samandoulgou., Cesar, J., Guerrini, L., Kabore-Zoungrana, C., \& Dulieu, D. (2007). Identification of ecological indicators for monitoring ecosystem health in the trans-boundary W Regional park: A pilot study. Biological Conservation, 138, 73-88.

Deckelbaum, R. J., Palm, C., Mutuo, P., \& DeClerck, F. (2006). Econutrition: Implementation models from Millenium Village Project in Africa. Food and nutrition Bulletin, 27 (4), 335-342.

Di Rienzo, J. A., Macchiavelli, R. \& Casanoves, F. (2010). Modelos lineales mixtos en Infostat. Grupo InfoStat, FCA, Universidad Nacional de Córdoba, Argentina. Recuperado el 30 de enero de 2013, de http://www.infostat.com.ar

Di Rienzo, J. A., Casanoves, F., Balzarini, M. G., Gonzalez, L., Tablada, M. \& Robledo, C. W. (2011). InfoStat versión 2011. Grupo InfoStat, FCA, Universidad Nacional de Córdoba, Argentina. Recuperado el 30 de enero de 2013, de http://www.infostat.com.ar

Harvey, C, A., Medina, A., Sánchez, D. M., Vílchez, S., Hernández, B., Saenz, J. C., Maes, J. M., Casanoves, F. \& Sinclair, F. L.(2006). Patterns of animal diversity in different forms of tree cover in agricultural landscapes. Ecological Applications, 16 (5), 1986-1999.

Hernández, S., Morales, S., Arendt, W, J. \& Tórrez, M. A. (2009). Bird Checklist Cerro Datanli-El Diablo Nature Reserve (1ra ed.). Managua: Jerry Bauer.

Holdridge, L. R. (1996). Ecología basada en zonas de vida. San José: Instituto interamericano de cooperación para la agricultura.

Howell, S.N. \& Webb, S. (1995). A guide to the birds of Mexico and the northern Central America. Oxford, New York: Oxford university Press.

IUCN. (2012). The IUCN red list of threatened species ${ }^{T M}$. Version 2012.2. Recuperado el 25 de noviembre de 2012, de: http://www.iucnredlist.org/.

Louman, B. \& Quirós, D. (Eds.). (2001). Silvicultura de bosques latifoliados húmedos con 
énfasis en América Central. Serie técnica (46), Turrialba: Centro Agronómico Tropical de Investigación y Enseñanza.

Maes, J. M. (2007). Identificación y Clasificación de Insectos de la Reserva Natural Datanlí-El Diablo. León: Informe de Consultoría para el Ministerio de los Recursos Naturales (MARENA).

Maes, J. M. (2013). Mariposas de Nicaragua. Recuperado el 29 de enero de 2013, de http://www.bio-nica.info/Biblioteca/BibliLepidoptera

Martínez-Sánchez, J. C. (2007). Lista Patrón de las aves de Nicaragua. (1 ed.). Managua: Alianza para las Áreas Silvestes.

Mas, A. H. \& Dietsch, T. V. (2004). Linking shade coffee certification to biodiversity conservation: butterflies and birds in Chiapas, Mexico. Ecological Applications, 14 (3), 642-654.

Moore, F. R., Gauthreaux, S. A., Kerlinger, P. \& Simons, T. (1995). Habitat requirements during migration important link in conservation. En T. E. Martin \& D. M. Finch. (Eds.) Ecology and management of neotropical migratory birds: a synthesis and review of critical issues, (pp. 121-144). New York: Oxford University Press.

Morales, H., Ferguson, B. G. \& García-Barrios, L. (2007). Agricultura: La cenicienta de la conservación en mesoamérica. En Harvey, C. A. \& Saénz, J. C. (Eds). Evaluación y conservación de Biodiversidad en paisajes fragmentados de Mesoamérica. (pp. 47-73). Santo Domingo de Heredia: Editorial Instituto Nacional de Biodiversidad.

Parra, J. L., Rahbek, C., McGuire, J. A. \& Graham, C. H. (2011). Contrasting patterns of phylogenetic assemblage structure along the elevational gradient for major hummingbirds clades. Journal of Biogeography, 38 (12), 2350-2361.

Pearman, P. B. (2002). The scale of community structure: habitat variation and avian guilds in tropical forest understory. Ecological Monographs, 72 (1), 19-39.

Pérez, A. M., Sotelo, M., Ramírez, F., Ramírez, I., López, A. \& Siria, I. (2006). Conservación de la biodiversidad en sistemas silvopastoriles de Matiguás y Río Blanco (Matagalpa, Nicaragua). Ecosistemas, (15), 125-141.

Philpott, S. M., Arendt, W. J., Armbrecht, I., Bichier, P., Diestch, T. V., Gordon, C., Greenberg, R., Perfecto, I., Reynoso-Santos, R., Soto-Pinto, L., Tejeda-Cruz, C., Williams-Linera, G., Valenzuela, J. \& Zolotoff, J. M. (2008). Biodiversity loss in Latin American coffee landscapes: Review of the evidence on ants, birds, and trees. Conservation Biology, 22 (5), 1093-1105.

Poulin, B., Lefebvre, G. \& McNeil, R. (1994). Diets of land birds from northeastern Venezuela. Condor, (96), 354-367.

Priess, J.A., Mimler, M., Klein, A.-M., Schwarze, S., Tscharntke, T. \& SteffanDewenter, I. (2007). Linking deforestation scenarios to pollination services and economic returns in coffee agroforestry systems. Ecological Applications, 17 (2), 407-417.

Remsen, J. V.,Jr., Hyde, M. A. \& Chapman, A. (1993). The diet of neotropical trogons, motmots, barbets, and toucans. Condor, (95), 178-192.

Steffan-Dewenter, I., Kessler, M., Barkman, J., Bos, M., Buchori., Erasmi, S., Faust, H., Gerold, G., Glenk, K., Gradstein, S. R., Guhardja, E., Harteveld, M., Hertel, D., Höhn, P., Kappas, M., Köhler, S., Leuschner, C., Maertensi, M., 
Marggraf, R., Migge-Kleian, Mogea, J., Pitopang, R., Schaefer, M., Schwarze, S., Sporn, S. G., Steingrebe, A., Tjitrosoedirdjo, S. S., Tjitrosoemito, S., Twele, A., Weber, R., Woltmann, L., Zeller, M. \& Tscharntke, T. (2007). Tradeoffs between income, biodiversity, and ecosystem functioning during tropical rainforest conversion and agroforestry intensification. PNAS, 104, 4973-4978. doi: 10.1073/pnas.0608409104.

Schulze, C. H., Walter, M., Kessler, J. A., Pitopang, R., Shahabuddin, Veddeler, D., Mühlenberg, M., Gradstein, S. R., Leushner, C., Steffan-Dewenter, I. \& Tscharntke, T. (2004). Biodiversity indicator groups of tropical land-use systems: comparing plants, birds, and insects. Ecological Applications, (14), 1321-1333.

Stiles, F. G. \& Skutch, A. F. (1989). A guide to the birds of Costa Rica. Utica, New York: Cornell University Press.

Stotz, D. F., Fitzpatrick, J. W., Parker, T. \& Moskovits, D. K. (1996). Neotropical birds: ecology and conservation. Chicago, Illinois: Chicago University Press.

Van Bael, S. A., Bichier P., Ochoa I. \& Greenberg R. (2007). Bird diversity in cacao farms and forest fragments of western Panama. Biodiversity and Conservation, (16), 224-2256.

Verea, C. \& Solórzano, A. (2001). La comunidad de aves del sotobosque de un bosque deciduo en Venezuela. Ornitologia Neotropical, (12), 235-253.

Vílchez, S., Harvey, C. A., Sánchez-Merlo, D., Medina, A., Hernández, B. \& Taylor, R. (2007). Diversidad y composición de aves en un agropaisaje de Nicaragua. En C. A. Harvey \& J. C. Saénz (Eds.). Evaluación y conservación de Biodiversidad en paisajes fragmentados de Mesoamérica, (pp. 547-576). Santo Domingo de Heredia: Editorial Instituto Nacional de Biodiversidad.

Walter, M., Bobo, K. S., Sainge, N. M., Fermon, H. \& Mühlenberg, M. (2005). From forest to farmland: Habitat effects on afrotropical forest bird diversity. Ecological Applications, (15), 1351-1366.

Willson, M. F., Porter, E. A. \& Condit, R. S. (1982). Avian frugivore activity in relation to forest light gaps. Caribbean Journal of Science, (18), 1-6. 